\title{
A 3-DIMENSIONAL ABRAHAM-SMALE EXAMPLE
}

\author{
CARL P. SIMON
}

\begin{abstract}
The author constructs an open set of non $\Omega$-stable diffeomorphisms on $T^{3}$.
\end{abstract}

To further the search for generic properties of diffeomorphisms, we construct a new counterexample to the genericity of Axiom A and $\Omega$ stable diffeomorphisms in $\operatorname{Diff}^{r}\left(T^{3}\right), r \geqq 1$. This paper reduces the dimension in the original counterexample of Abraham and Smale [1] by applying a technique of Williams [3]. For a general reference, see [2].

Let $g_{0}: T^{2} \rightarrow T^{2}$ be a hyperbolic automorphism with fixed point $\theta$. Let $g_{t}$ for $t \in[-1,+1]$ be a smooth isotopy of $g_{0}$ to "derived-from-Anosov" (D-A) maps $g_{-1}$ and $g_{1}$ as constructed in ([2], [3]). $g_{ \pm 1}(\theta)=\theta$, but $\theta$ is a source for $g_{+1}$ with 2-dimensional $W^{u}\left(\theta, g_{1}\right)$ and a sink for $g_{-1}$. The nonwandering set $\Omega\left(g_{1}\right)=\{\theta\} \cup \Sigma_{1}$, where $\Sigma_{1}$ is a 1-dimensional attractor; and $\Omega\left(g_{-1}\right)=\{\theta\} \cup \Sigma_{-1}$, where $\Sigma_{-1}$ is a 1-dimensional source. $T^{2}=W^{u}\left(\theta, g_{1}\right) \cup \Sigma_{1}$ $=W^{s}\left(\theta, g_{-1}\right) \cup \Sigma_{-1}$. Let $U, V$ be 2-disks in $T^{2}$ with $\theta \in$ int $U \subset \bar{U} \subset$ int $V \subset W^{u}\left(\theta, g_{1}\right) \cap W^{s}\left(\theta, g_{-1}\right)$ and with $g_{ \pm 1}^{ \pm 1}(U) \supset V$.

Using $g_{t}$, construct diffeomorphism $\bar{G}$ of $T^{2} \times[-1,1]$ such that (i) $T^{2} \times\{ \pm 1\}$ and $\{\theta\} \times[-1,1]$ are invariant under $\bar{G}$, (ii) $\bar{G} \mid T^{2} \times\{ \pm 1\}=g_{ \pm 1}$, (iii) $\bar{G}$ maps points in $U \times[-1,1]$ closer to $T^{2} \times\{+1\} \equiv T_{+1}^{2}$ and sends points outside $V \times[-1,1]$ closer to $T^{2} \times\{-1\} \equiv T_{-1}^{2}$. The $W^{u}\left(x_{+1}\right)$ and $W^{s}\left(x_{-1}\right)$ are now 2-dimensional for $x_{+1} \in \Sigma_{+1}$ and $x_{-1} \in \Sigma_{-1}$. Construct $\bar{G}$ so that for some such $x_{+1}$ and $x_{-1}, W^{u}\left(x_{+1}\right)$ and $W^{s}\left(x_{-1}\right)$ have a point of transversal intersection. (Alternatively, follow $\bar{G}$ by a map like $b$ below which forces a $W^{u}\left(x_{+1}\right)$ to intersect some $W^{s}\left(x_{-1}\right)$ transversely.) Since $\Sigma_{ \pm 1}$ are topologically transitive, we can write $\Sigma_{-1} \ll \Sigma_{+1}$ as in [1]. Extend $\bar{G}$ to a diffeomorphism of $T^{2} \times S^{1}$ with $(\theta, \pm 1) \equiv \theta_{ \pm 1}$ and $\Sigma_{ \pm 1}$ still hyperbolic. By composing $\bar{G}$ with a $C^{\infty}$ perturbation with support in $B=[V \backslash U] \times$ $(-1-\delta,-1+\delta)$, one easily obtains $G^{\prime}$ whose 2 -dimensional $W^{s}\left(\theta_{-1}, G^{\prime}\right)$ intersects the 1-dimensional unstable manifolds of $\Sigma_{-1}$ transversally in $B$.

Received by the editors August 31, 1971.

AMS 1970 subject classifications. Primary 58F20, 58F10.

Key words and phrases. Differentiable dynamical system, generic property, Axiom A, $\Omega$-stability.

${ }^{1}$ This work was partially supported by N.S.F. Grant GP-8007. 
Now, the 1-dimensional stable manifolds from $\Sigma_{+1}$ foliate $T_{+1}^{2} \backslash\left\{\theta_{+1}\right\}$. Let $G=b \circ G^{\prime}$ where $b$ has support in $V \times(1-\delta, 1+\delta)$ for small $\delta$ and forces the 1-dimensional $W^{u}\left(\theta_{-1}, G^{\prime}\right)$ to intersect the 2-dim set $\bigcup\left\{W^{s}(x)\right.$ : $\left.x \in \Sigma_{+1}\right\}$ transversally. Since $W^{u}\left(\Sigma_{+1}, G\right)_{d} \AA W^{s}\left(\Sigma_{-1}, G\right) \neq \varnothing, W^{u}\left(\Sigma_{-1}, G\right)_{\mathcal{L}}$ $W^{s}\left(\theta_{-1}, G\right) \neq \varnothing$, and $W^{u}\left(\theta_{-1}, G\right)_{1} \hbar W^{s}\left(\Sigma_{+1}, G\right) \neq \varnothing$, we have a cycle in $\Omega$. Since $\Sigma_{+1}$ has type $(1,2)$ and $\theta_{-1}$ has type $(2,1), G$ does not satisfy Smale's Axiom A [1]. Since the $W^{u}$ and $W^{s}$ vary continuously and the above intersections are transversal (at least, topologically), all maps sufficiently near $G$ will not satisfy Axiom A. The fact that none of these maps are $\Omega$-stable follows as in $\S 3$ of [1]. For, the set of $H^{\prime}$ such that the "first" intersection of $W^{s}\left(\theta_{+1}, H^{\prime}\right)$ with $W^{u}\left(\Sigma_{-1}^{\prime}, H^{\prime}\right)$ occurs on $W^{u}\left(x^{\prime} ; H^{\prime}\right)$ for some periodic $x^{\prime}$ in $\Sigma_{-1}^{\prime}$ and the set of $H^{\prime \prime}$ where the first such intersection occurs at a nonperiodic $W^{u}\left(x^{\prime \prime}, H^{\prime \prime}\right)$ with $x^{\prime \prime} \in \Sigma_{-1}^{\prime \prime}$ are both dense in a neighborhood of $G$. But no map in the former set can be $\Omega$-conjugate to any map in the latter set.

\section{REFERENCES}

1. R. Abraham and S. Smale, Nongenericity of $\Omega$-stability, Proc. Sympos. Pure Math., vol. 14, Amer. Math. Soc., Providence, R.I., 1970, pp. 5-8. MR 42 \#6867.

2. S. Smale, Differentiable dynamical systems, Bull. Amer. Math. Soc. 73 (1967), 747817. MR 37 \#3598.

3. R. F. Williams, The D-A maps of Smale and structural stability, Proc. Sympos. Pure Math., vol. 14, Amer. Math. Soc., Providence, R.I., 1970, pp. 329-334. MR 41 \#9196.

Department of Mathematics, University of California, Berkeley, California 94720

Current address: Department of Mathematics, University of Michigan, Ann Arbor, Michigan 48104 